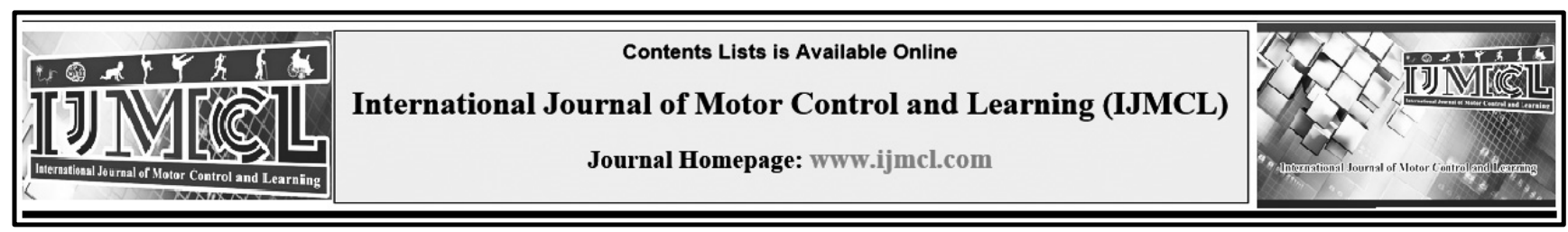

\title{
Comparison the Effect of High and Low Contextual Interference Training on Performance Accuracy
}

\author{
Zeinab Shenasa $^{a}$, Robabeh Rostami ${ }^{b^{*}}$, Gholamhosein Nazemzadehgan $^{c}$, Bahman Hajaatnia $^{d}$
}

${ }^{a}$ M.Sc. of Motor Behavior, Shiraz University, Shiraz, Iran

${ }^{\mathrm{b}}$ Associate Prof. of Motor Behavior, Shiraz University, Shiraz, Iran

${ }^{\mathrm{c}}$ Assistant Prof. of Motor Behavior, Shiraz University, Shiraz, Iran

${ }^{\mathrm{d}}$ Ph.D. of Health Psychology, Islamic Azad University South Tehran Branch, Tehran, Iran

\begin{tabular}{l}
\hline Keywords \\
\hline Exercise \\
Contextual Interference \\
Accuracy \\
Performance
\end{tabular}

Robabeh Rostami,

Email: rostami@shirazu.ac.ir

Received: 2020/07/10

Accepted: 2020/10/14

Published: 2020/11/05

\begin{abstract}
Objective: The aim of this study was to determine the differences between different methods of contextual interference (High and Low) on accuracy.

Methods: The subjects were 51 people from Shiraz Mental Health Park Rehabilitation Center who were purposefully selected and after the pre-test, they were randomly divided into three groups. The High and Low interference groups performed 180 Frisbee attempts (backhand, forehand, and hammer) and the control group played a team game at the same time as the intervention groups. Then, acquisition, retention and immediate transfer tests were taken. Accuracy was measured using a researcher-made test.
\end{abstract}

Results: The results of repeated measures test showed no significant difference in the phase of acquisition and there was a significant difference between the groups in the phases of transfer $(\mathrm{P}=0.011)$ and retention $(\mathrm{P}=0.026)$.

Conclusion: It seems that the results of this study can be effective in reducing researchers' concerns in simultaneous training of several motor skills by emphasizing the movement pattern and achieving the goal of movement (accuracy). Findings indicate that the use of contextual interference methods, at high levels, leads to time savings and stabilizes the pattern of movement in the learning phases.

\section{Introduction}

Practice and more importantly, planning training sessions are known as very important variables in the field of motor behavior. The importance of the training variable affects other variables, and it is very important to mention it in all situations. If the other conditions are the same, more learning will result from more repetitions (Schmidt \& Lee, 2009). Training conditions are one of the most influential factors in training and learning motor skills. The instructor must organize the practice of the various skills in specific ways.
Using the effect of contextual interference is a way to create this effective and dynamic training environment. Contextual interference is defined as interference in learning and performance, and occurs when one task is practiced during another task. Blocked training (low interference) is a type of training method in which the learner completes all the training efforts before starting the next task. On the other hand, in random training (high interference), the order of presentation of tasks and skills has a random arrangement, so different skills are combined during practice (R. Magill \& 
Anderson, 2010). Many studies that have examined the effect of contextual interference have examined the acquisition of skills following a random order of training efforts compared to the practice of homework through blocked efforts (Jared M Porter \& Saemi, 2010). Some of these studies have supported the effect of contextual interference and have shown the superiority of randomized exercise over blocking(She and Wright, 2001; Schmidt, 2002; Claire, Lee, Weiss \& Relia, 2006; Kitch \& Lee, 2007), But some research has not supported the effect of contextual interference(Sears and Hosak, 1987; Bortoli, Robaza, Dorigon and Kara, 1992; Mira and Tani, 2001; Jones and Frank, 2007; Russell and Newell, 2007; Sheikh 2012).

Jork (1990 and 1994), in a favorable difficulty perspective, states that increasing difficulty during practice leads to increased retention and long-term transfer. In order for the learner to take advantage of the desired difficulties, he must be challenged at the appropriate levels. To this end, he states that the levels of contextual interference must be combined (Jared Marak Porter, 2008). In addition to Jork (1990 and 1994), Gadagnoli and Lee (2004) have proposed the challenge hypothesis. Based on the challenge hypothesis, programs with low levels of background interference (blocked practice) are more useful for people with lower levels of skill than training programs with high levels of background interference (random training). They stated that the appropriate challenge during training provides an optimal learning environment (OnlaOr \& Winstein, 2008). One way to progressively create the right challenge as the learner's skill level grows is to use a gradual increase in contextual interference (Jared Marak Porter, 2008).

Exercise is often considered as an effective factor in the development of motor skills (Adams, 1964; Annette, 1966; Schmidt \& Lee, 1999; Megil, 2001), Because if all factors are kept constant, skill development is significantly related to the amount of practice (Crossman, 1959; Newell \& Rosonbaum, 1981) (Azizi, Saberi Kakhaki, \& Daneshvar, 2009).

One of the first aspect of measurement of such features in motor behavior is quantifying the extent to which a movement has achieved a desired or learned goal. Motor functions are complex, and measuring their accuracy requires more knowledge and experience (Schmidt \& Lee, 2015).

Contemporary neurological models recognize addiction as a brain disorder that includes: Severe neuronal damage and leads to substance abuse despite its negative consequences (Moreno-López et al., 2012).

Various sports, including Frisbee, can affect people's accuracy. Frisbee is an exciting sport that can be performed in most environments and is designed based on simple techniques (Majdara, 2013). Frisbee was chosen because people had little experience in the sport and the skills used in this study were new skills that people were not familiar with before.

People practice skills to increase their ability to implement skills in the future. Exercise variability is one of the characteristics of exercise that increases a person's likelihood of success, and is defined as the variety of movements or contextual characteristics that a person experiences during 
exercise, this raises the question, what is the most effective and efficient method of training? One of the characteristics of practice that increases the probability of success in future performance is variability in learning practice experiences. The first step in determining the amount of variability is to estimate the performance characteristics of the future (Richard A. Magill, 2001).

The main findings of the study, entitled "Contextual Interference Effects on Motor Skill Acquisition, Aetention and Transfer in Sport Rifle Shooting", are that practice enhanced shooting accuracy. Accuracy between groups during the acquisition phase, retention test results, and transfer test results were similar between groups. Therfor, contextual interference effect was not observed in any of the phases of motor learning in sport rifle shooting (Moretto, Marcori, \& Okazaki, 2018). In a study entitled "The Comparison of the effect of various levels of contextual interference (blocked, random and incremental) on acquisition, retention and transmission of movment time error and cursor error in complex motor- perceptual task, achieved this result that there was no significant difference between the three groups in acquisition, retention and transfer (Sheikh, 2014).

But, in a study entitled Effects of Contextual Interference on Feeding Training in Patients with Strokes, concluded that: Random practice was more effective in learning feeding skills in patients with stroke than blocked practice (especially in the transition phase), and in general, it has been shown that random practice is more effective than blocked practice (Jo, Noh, \& Kam, 2020). Also, in another study entitled The effect of exercises with contextual interference of training on attention problems in children with developmental coordination disorder, concluded that: Exercises with more contextual interference can further improve attention problems in children with developmental coordination disorders (Moradi, Sohrabi, \& Mones Tusi, 2017).

The main findings of the study entitled "Exploring contextual interference in implicit and explicit motor learning" shown that contextual interference helps to retain information for longer periods of time that can more easily be accessed later on. however, there is no guarantee that the information is completely accurate. this shows that experiencing interference is more beneficial for motor tasks where the primary goal is selecting an action plan as fast possible (Dang, Parvin, \& Ivry, 2019).

The results of some researches sometimes contradictorily point to the superiority of each of the blocked or random methods, and since this type of research is a continuous overlap between two parties, it is necessary to design exercises that are more capable in terms of the degree of contextual interference, especially in people whose processing part is disturbed. Therefore, considering the incremental method of contextual interference training in this study has been examined to answer the question of whether it is possible to use these training methods (upper or lower contextual interference) to Did the process of improving the accuracy of addicts help or not? 


\section{Method}

The present study was pseudo-experimental and the research design consisted of three groups (high field interference training group, low background interference training group and control group) with measurement steps (acquisition, transfer and memorization).

\section{Subjects}

The statistical population of the present study included addicts living in the treatment and rehabilitation center of the mental health park (Salami village), Purposeful and voluntary sampling was selected based on the criteria of age, time of arrival at the center, type of drug use (traditional, industrial and semi-industrial) and type of addiction and after the pretest, they were divided into three groups of 17 people (high interference, low interference and control). Group tags were determined randomly.

\section{Apparatus}

In this study, data were collected in two parts. First: The personal information of the subjects including: date of birth, height, weight, body mass index (BMI), age of onset of addiction, type of substance, duration and history of use and treatment of opioids were recorded in the demographic form. In the following, a tool was developed according to the objectives of the study (Frisbee skill accuracy test), that only measured accuracy. This test was designed and performed with modifications from Zipp and Gentile (2010) research. The reliability of the researcher-made accuracy measurement tool using the test method, retest (40 people), was equal to 0.94 . To perform the test, the subject stood at a distance of 6 meters from a ring with a diameter of $85 \mathrm{~cm}$ and at a height of one and a half meters above the ground and threw a Frisbee disc towards the ring. The scoring method of this test was as follows:

Frisbee disk passes through the ring without collision and directly $=3$ points.

Place the Frisbee disc inside the ring by hitting the sides of the ring $=2$ points.

The Frisbee disc should not be placed inside the ring by hitting the sides of the ring $=1$ point.

Frisbee disc go out of the ring without colliding = 0 points.

\section{Experimental Designs}

Due to the fact that doing research on addicts is a very difficult and requires special permits from relevant organizations, so at the beginning of the work to get the code of ethics (IR.SUMS.REC.1397.518) from Shiraz University of Medical Sciences and also obtained a license from the General Department of Welfare of Fars Province. Then, by examining the addiction file, length of stay and dose of addicts under treatment, In coordination with the director of the center, informing the addicts under treatment, after explaining the current plan and the benefits of participating in the plan, individuals were asked to participate in this plan voluntarily. Then the consent form and biography questionnaire (personal information) were completed by individuals. In the next step, the height and weight of the individuals were measured by the researcher and they were shown selected Frisbee skills. After 
that, the subjects performed three attempts of the desired skills (backhand, forehand and Hammer) and then participated in the Frisbee accuracy skill pre-test and the scores were recorded. Based on the pre-test scores, individuals were matched in three equal groups of 17 people (high-interference training group, low-interference training group and control group), The research groups were named by random assignment. In the next session, the subjects practiced Frisbee skills according to the contextual intervention training program related to their group, which performed a total of 180 throws in both groups, and at the end of the acquisition tests, immediate transfer (after the acquisition test and with Change of throw angle) and retention (24 hours after immediate transfer test) were taken. It should be noted that the implemented protocol for pre-test, delayed retention and transfer was selected based on the research protocol of Deakin and Proteau (2000) and Wright, Li, and Coady (1997). The control group played a team game with the ball at the same time as the intervention groups. In this study, contextual interference means the application of high and low interference in Frisbee throwing skills such as (forehand, backhand and Hammer). The purpose of the high interference practice was to perform the throws in the form of 5 blocks of 6 ( 3 sets per day) and the blocks in the form of: 1- Backhand, Forehand, Hammer. 2Forehand, Backhand, Hammer. 3- Forehand, Hammer, Backhand. 4- Backhand, Hammer, forehand. 5- Hammer, forehand, Backhand. 6Hammer, Backhand, forehand. Was performed and the purpose of the exercise with low interference was to perform the actions of the throws in the form of 5 blocks of 6 ( 3 sets per day) and the blocks in the form of: 1- Backhand, Backhand, Backhand. 2Forehand, forehand, forehand. 3- Hammer, Hammer, Hammer. 4- Backhand, Backhand, Backhand. 5- Forehand, forehand, forehand. 6Hammer, Hammer, Hammer, was done. In both groups, they had 5 seconds to throw for each attempt, and they had a 5-second break between these 6 blocks, and the rest between the blocks was 2 minutes. And the control group, at the same time as the intervention groups, performed a similar exercise to team play (throwing and receiving the ball).

\section{Data Analysis}

In this study, descriptive statistics indices were used to analyze the collected demographic variables, plotting the graphs, and drawing the tables in order to analyze the collected information. The Shapiro-Wilk test was used to determine the normality of the study data. To answer the research questions, repeated measurement tests and oneway analysis of variance were used. Excel 2016 software was used to draw the figures. Statistical operations were performed by SPSS software version 22 and the significance level of the tests was set at 0.05 .

\section{Results}

Descriptive information of the demographic variables of the subjects is presented in Table (1). Descriptive characteristics of the steps of measuring the dependent variable in research groups are presented in Table (2). 
Table 1. Descriptive information of the demographic variables of the subjects.

\begin{tabular}{ccccccc}
\hline Groups & Qty & $\begin{array}{c}\text { Age (Years) } \\
(\mathbf{M} \pm \mathbf{S D})\end{array}$ & $\begin{array}{c}\text { Height }(\mathbf{c m}) \\
(\mathbf{M} \pm \text { SD) }\end{array}$ & $\begin{array}{c}\text { Weight }(\mathbf{K g}) \\
(\mathbf{M} \pm \text { SD) }\end{array}$ & $\begin{array}{c}\text { Body Mass } \\
\text { Index } \\
(\mathbf{B M I}) \\
(\mathbf{M} \pm \mathbf{S D})\end{array}$ & $\begin{array}{c}\text { Methadone dose } \\
(\mathbf{m l}) \\
(\mathbf{M} \pm \mathbf{S D})\end{array}$ \\
\hline $\begin{array}{c}\text { High } \\
\text { Interference }\end{array}$ & 17 & $29.3 \pm 47.92$ & $172.6 \pm 71.35$ & $57.8 \pm 59.97$ & $19.2 \pm 24.31$ & $19.1 \pm 71.634$ \\
\hline $\begin{array}{c}\text { Low } \\
\text { Interference }\end{array}$ & 17 & $29.4 \pm 12.40$ & $174.7 \pm 18.44$ & $62.8 \pm 12.65$ & $20.2 \pm 44.26$ & $18.1 \pm 82.635$ \\
\hline Control & 17 & $29.3 \pm 88.68$ & $169.8 \pm 12.21$ & $56.5 \pm 24.04$ & $19.2 \pm 73.09$ & $21.1 \pm 94.038$ \\
\hline
\end{tabular}

Table 2. Descriptive characteristics of the steps of measuring the dependent variable in research groups.

\begin{tabular}{cccc}
\hline Levels & \multicolumn{1}{c}{ Group } & Mean & The standard deviation \\
\hline \multirow{3}{*}{ Pre-Test } & Low interference & 13.88 & 7.052 \\
& Control & 10.82 & 5.491 \\
\cline { 2 - 3 } Post-test (acquisition) & High interference & 11.41 & 6.001 \\
\cline { 2 - 3 } & Low interference & 14.24 & 5.056 \\
\cline { 2 - 3 } Retention & Control & 15.06 & 3.473 \\
\hline & High interference & 16.94 & 3.881 \\
\hline & Low interference & 17.53 & 5.088 \\
\hline & Control & 16.06 & 3.250 \\
\hline Transfer & High interference & 22.00 & 3.500 \\
\hline & Low interference & 11.65 & 3.790 \\
\hline & Control & 10.94 & 4.815 \\
\hline
\end{tabular}

The hypothesis of normality of data was measured by Shapirovilk test. According to Shapirovilk test, the hypothesis of normality for data in different sessions and research groups is confirmed. In order to use the statistical test of analysis of variance in duplicate measurements, the sphericity hypothesis was confirmed by Mauchly's test $(\mathrm{P}=0.523)$.

Given that our research design was both WithinSubjects and Between-Subjects, Analysis of variance with repeated measures was used, And the results showed that there is a significant difference between the groups $(\mathrm{F}=7.236$, $\mathrm{Sig}=0.002$, Eta $=$ 0.232 and $\mathrm{Df}=2$ ). It also showed that there is a significant difference between the stages $(\mathrm{F}=$ 22.099, $\mathrm{Sig}=0.000, \mathrm{Eta}=315$ and $\mathrm{Df}=3$ ).

Levin test was used for homogeneity of variances and the results of this test showed that the assumption of homogeneity of variances for acquisition $(\mathrm{P}=0.537)$, transfer $(\mathrm{P}=0.246)$ and retention $(\mathrm{P}=0.473)$ was established.

One-way analysis of variance (ANOVA) with a significance level of $\mathrm{P}<0.05$ was performed to identify the intergroup difference. The results of
ANOVA research showed that there was no significant difference between the pretest $(\mathrm{F}=$ $1.159, \mathrm{Sig}=0.322$ and $\mathrm{Df}=2$ ), In the post-test neighborhood, there is no significant difference between the groups $(F=1 / 862$, Sig $=0.166$ and $D f$ $=2)$. In the retention phase, a significant difference was observed between the groups $(\mathrm{F}=689 / 8$, Sig $=$ 0.001 and $\mathrm{Df}=2)$, Also, in the transition phase, a significant difference was observed between the groups $(\mathrm{F}=14.958, \mathrm{Sig}=0.000$ and $\mathrm{Df}=2)$.

To identify the two stages in which the groups differed, Tukey post hoc test was used, The results showed that in the retention phase, Low interference is significantly different from high interference $(\mathrm{Sig}=0.011$ ), And there is a significant difference between the control group and high interference (Sig $=0.001$ ), In the transition phase, there is a significant difference between high and low interference $(\mathrm{Sig}=0.000)$, There is also a significant difference between the control group and high interference $(\mathrm{Sig}=0.000)$. 


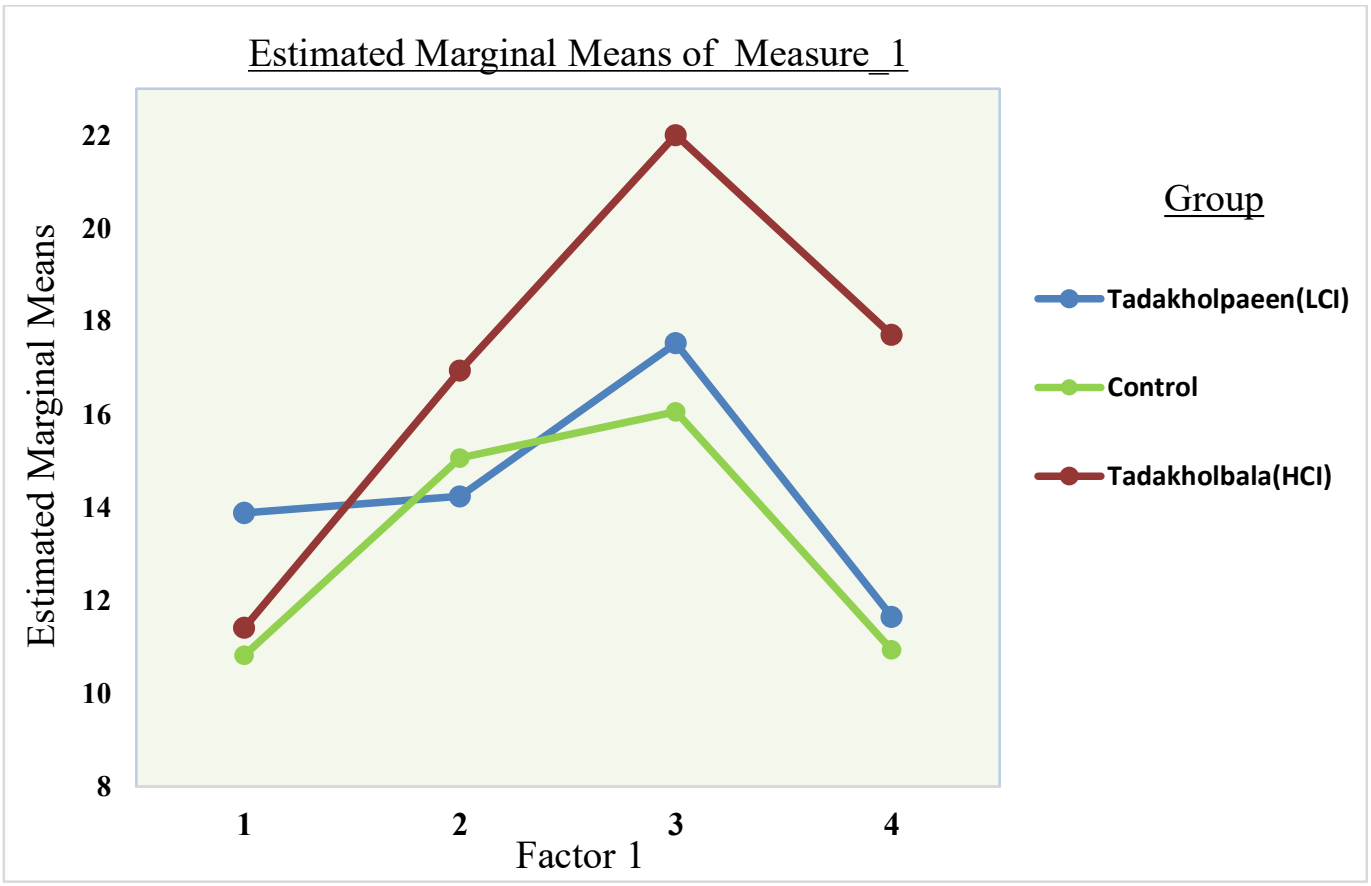

Figure 1. Estimated Marginal Means of Measure 1 in different groups.

Then the repeat measurement test was performed for the Low Contextual Interference (LCI) group and the results showed that there was a significant difference between the stages $(\mathrm{F}=5.341$, $\mathrm{Sig}=$ 0.003, Eta $=250$ and Df $=3$ ), Using Bonferroni test, the location of the difference in the low interference between the retention phase and the transfer was determined $(\mathrm{Sig}=0.001)$, These steps were also performed for the High Contextual Interference (HCI) Group and the control group. The results showed that in the upper control group, there was a significant difference between the stages $(\mathrm{F}=19.49, \mathrm{Sig}=0.000, \mathrm{Eta}=549$ and $\mathrm{Df}=$ 3), And using Bonferroni test, the place of difference in high interference between pre-test and acquisition stages ( $\mathrm{Sig}=0.05)$, acquisition and retention $(\mathrm{Sig}=0.000)$, and retention and transfer $(\mathrm{Sig}=0.000)$, the results also showed that in the control group, there is a significant difference between the stages $(\mathrm{F}=6.455$, $\mathrm{Sig}=0.001$, Eta $=$
0.287 and $\mathrm{Df}=3$ ), and using Bonferroni test, the place of difference in the control group, between the pre-test and acquisition stages ( $\mathrm{Sig}=0.045)$, and retention and transfer $(\mathrm{Sig}=0.029)$, significant changes seen in the control group (The control group has a pattern similar to the low interference group), it can be related to research limitations. Because everyone lived in the same environment and saw the efforts, tests, and exercises of other groups, and they modeled. These effects may be due to these cases.

Therefore, high-interference training, even in people who are addicted and in the rehabilitation period, can lead to better learning and better performance than low-interference in all stages of learning (acquisition, retention, and transfer). and the practical recommendation of this research is that: if these people want to do something that requires precision, they can use high-interference practice to improve accuracy. 


\section{Discussion and Conclusion}

The aim was to determine the differences between different interference methods (high and low) on the performance accuracy. For this purpose, interference methods were used in throwing skills of Frisbee sport. Exercise planning in motor behavior is a new method in training. Contextual interaction, using a wide variety of cognitive and motor abilities, has recently found its place in the development of motor skills. The results of the present study showed that there is no significant difference between high and low contextual interference on accuracy in the acquisition session of Frisbee skill. No research was found in regards with the main purpose of the present study. However, the results of this study are the same as the results of Arab-Ameri, Hemayattalab, Karimiyani, and Sami (2012), which measured the effect of basketball training methods on acquisition, retention and transfer, in the acquisition phase between groups. Feghhi, Abdoli, and Valizadeh (2011) also showed that the method of gradually increasing the contextual interference is useful for learning a separate task. And confirms the results of the present study, which at the acquisition session did not differ significantly between high and low interference

The results of this study are not the same as the research of Feghhi, Valizadeh, Rahimpour, Tehrani, and Karampour (2015), Cheldavi, Shakerian, Boshehri, and Zarghami (2014), Tsai, Wang, and Tseng (2012), Penkman (2004), Rommel, Halperin, Mill, Asherson, and Kuntsi (2013). The reasons for the discrepancy include items such as different subjects, how to take the test and the duration of the research. So, in general, it can be said that training sequences in which task situations are deliberately changed from one effort to another are relatively more effective for adults and much more effective for children than fixed training situations. Random practice destroys performance, but facilitates transfer and retention (Salman, 2015).

The results of the present study showed that there is a significant difference between high and low contextal interference on accuracy in the transfer phase of Frisbee skills. The results from the retention and transfer test support the theory of Richard A Magill and Hall (1990) and the Guadagnoli and Lee (2004) challenge point hypothesis. No research was found in regards with the main purpose of the present study. However, the results of Moradi et al. (2017), (Cheldavi et al., 2014), Tsai et al. (2012), Rommel et al. (2013), Jared M Porter and Saemi (2010) have confirmed the results of our research.

Also in the study of Dias and Mendes (2010), the effect of gradually increasing the contextual interference on golf learning was investigated, the results of this study are in coflict with our research. Rasons for this conflict can be the type and duration of sports activities, gender and individual differences. In addition, in their study, Prado et al. (2017) concluded that high-level contextual interference (random) in the transfer session, in both groups of people with cerebral palsy and developing people, performed better than it showed low contextual interference (blocked) exercises.

The results of the present study showed that there is a significant difference between high and low 
contextual interference on the accuracy of Frisbee skill retention session. No research was found in regards with the main purpose of the present study. However, the results of the researches of Moradi et al. (2017), Feghhi et al. (2015), Cheldavi et al. (2014), Pauwels, Swinnen, and Beets (2014), Tsai et al. (2012), Rommel et al. (2013) , Have confirmed the results of the present study. The most important reason for alignment is the type of exercise (all of the above research on the contextual interference training method).

Also, the results of the research of Ghasem Karimi, Mohammadzadeh Jahatlo, and Hosseini (2013), entitled The effect of transfer training arrangement on contextual interference in learning badminton skills (long service, defensive dice, backhand dice), showed that in The instant retention test, only the difference between the blocked and random groups with the blocked superiority, was significant and did not confirm the results of the present study, but in the delayed retention test both random and transferable groups performed significantly better than the blocked group. These results confirm the results of the present study.

In addition, the research of Dias and Mendes (2010), Jones and French (2007) does not confirm the results of our research. Dias and Mendes examined the effect of a gradual increase in contextual interference on golf learning, while Jones and French examined the effect of interference training on volleyball players.

Salehi, Khajeh, and NAMAZI (2010), in a study entitled "Regular increase of contextual interference in learning basketball passes", concluded that to facilitate the learning of several motor skills, the arrangement of sessions should start from low interference and plan towards high interference. Research results of Shafizadeh and Shaban (2018) as the effect of contextual interference on acquisition, retention and transfer of childrens basic gymnastics skills, confirmed the superiority of random exercise in the age range of three to six years in gymnastics and emphasized the effect of skill type on contextual interference .

Thürer, Gedemer, Focke, and Stein (2019) in a study entitled: Contextual interference effect is independent of retroactive inhibition but variable practice is not always beneficial, concluded that the Random group showed better consolidation performance compared to both Blocked groups, and no benefits in absolute performance values. This indicates that CIE reflects a true motor learning phenomenon, which is independent of retroactive inhibition. However, random practice is not always beneficial over constant practice.

Based on these results, it can be suggested that educators working in treatment and rehabilitation centers related to addicts under treatment, can use high contextual interference exercises in sport like Frisbee, to improve accuracy as well as performance and learning. In addition, the use of contextual interference methods, even at high levels, saves time and stabilizes the movement pattern in the learning process.

\section{Acknowledgments}

The authors of this article would like to express their gratitude and appreciation to all the addicts treated by the Mental Health Park Treatment and Rehabilitation Center (Salami Village) who 
participated in this study, and without their participation, this study would not have been possible.

\section{References}

1. Arab-Ameri, E., Hemayattalab, R., Karimiyani, N., \& Sami, S. (2012). Effect of Blocked, Random, and systematically Increasing Practice Schedules on Learning of Various Basketball Pass. JRSM, 1(2), 87-99.

2. Azizi, S., Saberi Kakhaki, A., \& Daneshvar, A. (2009). Comparison of the effect of different levels of contextual interference (High and Low) on the acquisition, retention and transfer of simple and complex navigation and click skills with the mouse in the initial learning and training courses. In (pp. 40-45). Dissertation. Shahid Bahonar University of Kerman.

3. Cheldavi, H., Shakerian, S., Boshehri, S. N. S., \& Zarghami, M. (2014). The effects of balance training intervention on postural control of children with autism spectrum disorder: Role of sensory information. Research in Autism Spectrum Disorders, 8(1), 8-14.

4. Dang, K. V., Parvin, D. E., \& Ivry, R. B. (2019). Exploring Contextual Interference in Implicit and Explicit Motor Learning. BioRxiv, 644211.

5. Deakin, J. M., \& Proteau, L. (2000). The role of scheduling in learning through observation. Journal of motor behavior, 32(3), 268-276.

6. Dias, G., \& Mendes, R. (2010). Efeitos do contínuo de níveis de interferência contextual na aprendizagem do" putt" do golfe. Revista Brasileira de Educação Física e Esporte, 24(4), 545-553.

7. Feghhi, I., Abdoli, B., \& Valizadeh, R. (2011). Compare contextual interference effect and practice specificity in learning basketball free throw. Procedia-Social and Behavioral Sciences, 15, 2176-2180.

8. Feghhi, I., Valizadeh, R., Rahimpour, M., Tehrani, M. A., \& Karampour, S. (2015). Contextual interference in learning three table tennis services. Procedia-Social and Behavioral Sciences, 191(2), 546-549.

9. Ghasem Karimi, S., Mohammadzadeh Jahatlo, H., \& Hosseini, F. (2013). Effect of Transitional Practice Schedule on Contextual Interference in Badminton Skills Learning (Long Service, Forehand Toss and Backhand Toss). Journal of Motor Learning and movment, 5(1), 221.

10. Guadagnoli, M. A., \& Lee, T. D. (2004). Challenge point: a framework for conceptualizing the effects of various practice conditions in motor learning. Journal of motor behavior, 36(2), 212-224.

11. Jo, E.-J., Noh, D.-H., \& Kam, K.-Y. (2020). Effects of contextual interference on feeding training in patients with stroke. Human Movement Science, 69, 102560.

12. Jones, L. L., \& French, K. E. (2007). Effects of contextual interference on acquisition and retention of three volleyball skills. Perceptual and motor skills, 105(3), 883-890.
13. Magill, R., \& Anderson, D. (2010). Motor learning and control: Concepts and Applications: McGraw-Hill Publishing.

14. Magill, R. A. (2001). Motor Learning: Concepts and Applications (M. K. Vaez Mousavi \& M. Shojaei, Trans.): Hannaneh Publication.

15. Magill, R. A., \& Hall, K. G. (1990). A review of the contextual interference effect in motor skill acquisition. Human Movement Science, 9(3-5), 241-289.

16. Majdara, A. (2013). Excercise For Everyone (Vol. 2): Avaye Zohor Pulication.

17. Moradi, H., Sohrabi, M., \& Mones Tusi, M. (2017). The effect of exercises with contextual interference of training on attention problems in children with developmental coordination disorder. Journal of Shahrekord Uuniversity of Medical Sciences, 19.

18. Moreno-López, L., Stamatakis, E. A., FernándezSerrano, M. J., Gómez-Rio, M., Rodríguez-Fernández, A., Pérez-García, M., \& Verdejo-García, A. (2012). Neural correlates of hot and cold executive functions in polysubstance addiction: association between neuropsychological performance and resting brain metabolism as measured by positron emission tomography. Psychiatry Research: Neuroimaging, 203(2-3), 214-221.

19. Moretto, N. A., Marcori, A. J., \& Okazaki, V. H. A. (2018). Contextual interference effects on motor skill acquisition, retention and transfer in sport rifle shooting. Human Movement, 19(2), 99-104.

20. Onla-Or, S., \& Winstein, C. J. (2008). Determining the optimal challenge point for motor skill learning in adults with moderately severe Parkinson's disease. Neurorehabilitation and neural repair, 22(4), 385-395.

21. Pauwels, L., Swinnen, S. P., \& Beets, I. A. (2014). Contextual interference in complex bimanual skill learning leads to better skill persistence. PloS one, 9(6), e100906.

22. Penkman, L. (2004). Remediation of attention deficits in children: A focus on childhood cancer, traumatic brain injury and attention deficit disorder. Pediatric Rehabilitation, 7(2), 111-123.

23. Porter, J. M. (2008). Systematically increasing contextual interference is beneficial for learning novel motor skills. 50-57.

24. Porter, J. M., \& Saemi, E. (2010). Moderately Skilled Learners Benefit by Practicing with Systematic Increases in Contextual Interference. International journal of coaching science, 4(2).

25. Prado, M. T. A., Fernani, D. C. G. L., da Silva, T. D., Smorenburg, A. R., de Abreu, L. C., \& de Mello Monteiro, C. B. (2017). Motor learning paradigm and contextual interference in manual computer tasks in individuals with cerebral palsy. Research in Developmental Disabilities, 64, 56-63.

26. Rommel, A.-S., Halperin, J. M., Mill, J., Asherson, P., \& Kuntsi, J. (2013). Protection from genetic diathesis in attention-deficit/hyperactivity disorder: possible complementary roles of exercise. Journal of the American Academy of Child \& Adolescent Psychiatry, 52(9), 900-910.

27. Salehi, H., Khajeh, F., \& NAMAZI, Z. M. (2010). Regular increasing contextual interference effect in 
learning of basketball passing. MOTOR BEHAVIOR (RESEARCH ON SPORT SCIENCE) 2(5), 107-126.

28. Salman, Z. (2015). Motion behavior Relationship mind and body with the goal of optimal performance: Hatmi Publication.

29. Schmidt, R. A., \& Lee, T. D. (2009). Control and learning Motor (A. Farokhi, A. Bahram, \& H. Khalaji, Trans. Vol. 1): Nersi publication.

30. Schmidt, R. A., \& Lee, T. D. (2015). Learning and motor function (R. Hamayat Talab \& A. Ghasemi, Trans. Vol. 2).

31. Shafizadeh, A., \& Shaban, E. (2018). The effect of contextual interference on acquisition, retention and transfer of childrens basic gymnastics skills. Motor Behavior, 10(31), 161-176.

32. Sheikh, M. (2014). The Comparison of the Effect of Various Levels of Contextual Interference (Blocked, Random and Increasing) on Acquisition, Retention and Transfer of Movement Time Error and Cursor Error in Complex Motor - Perceptual Task. Journal of Motor Learning and Movement, 6(3), 293-308. doi:10.22059/jmlm.2014.51870

33. Thürer, B., Gedemer, S., Focke, A., \& Stein, T. (2019). Contextual interference effect is independent of retroactive inhibition but variable practice is not always beneficial. Frontiers in human neuroscience, 13, 165.

34. Tsai, C.-L., Wang, C.-H., \& Tseng, Y.-T. (2012). Effects of exercise intervention on event-related potential and task performance indices of attention networks in children with developmental coordination disorder. Brain and cognition, 79(1), 12-22.

35. Wright, D. L., Li, Y., \& Coady, W. (1997). Cognitive processes related to contextual interference and observational learning: A replication of Blandin, Proteau, and Alain (1994). Research quarterly for exercise and sport, 68(1), 106-109.

36. Zipp, G. P., \& Gentile, A. (2010). Practice schedule and the learning of motor skills in children and adults: teaching implications. Journal of College Teaching \& Learning (TLC), 7(2). 